\title{
Concentrated sugars and incidence of prostate cancer in a prospective cohort
}

\author{
Fayth L. Miles ${ }^{1,2 *}$, Marian L. Neuhouser ${ }^{2}$ and Zuo-Feng Zhang ${ }^{1,3,4}$ \\ ${ }^{1}$ Department of Epidemiology, Fielding School of Public Health, University of California, Los Angeles, CA 90095-1772, USA \\ ${ }^{2}$ Cancer Prevention Program, Division of Public Health Sciences, Fred Hutchinson Cancer Research Center, Seattle, WA \\ 98109, USA \\ ${ }^{3}$ David Geffen School of Medicine, Center for Human Nutrition, UCLA, Los Angeles, CA 90095, USA \\ ${ }^{4} J o n s s o n$ Comprehensive Cancer Center, UCLA, Los Angeles, CA 90095-1781, USA
}

(Submitted 6 November 2017 - Final revision received 17 May 2018 - Accepted 18 May 2018 - First published online 26 July 2018)

\section{Abstract}

The association between consumption of added or concentrated sugars and prostate cancer risk is unclear. We examined the association between concentrated sugars in beverages and desserts and prostate cancer risk among 22720 men in the usual-care arm of the Prostate, Lung, Colorectal and Ovarian (PLCO) Cancer Screening Trial, enrolled during 1993-2001. After a median follow-up of 9 years, 1996 men were diagnosed with prostate cancer. Cox proportional hazards regression models were used to estimate hazard ratios (HR) for prostate cancer risk and $95 \% \mathrm{CI}$, adjusting for potential confounding factors. Increased consumption of sugars from sugar-sweetened beverages was associated with increased risk of prostate cancer for men in the highest quartile of sugar consumption (HR: 1.21; 95\% CI 1.06, 1.39), and there was a linear trend $(P<0.01)$. There were no linear associations between prostate cancer risk and consumption of sugars from fruit juices or dessert foods. In conclusion, in this prospective substudy within the PLCO trial, consumption of sugars from sugar-sweetened beverages was associated with increased risk of prostate cancer among men receiving standard medical care. Our study suggests that limiting intake of sugars from beverages may be important in the prevention of prostate cancer.

Key words: Added sugars: Proportional hazards regression: Prostate cancer risk: Prospective cohorts

Consumption of added sugars in America has increased considerably over time ${ }^{(1)}$. Dietary intake of energetic sweeteners including sucrose and high-fructose maize syrup increased by nearly $40 \%$ between $1950-59$ and $2000^{(2)}$. Consumption of added sugars from beverages, particularly, has increased. Between 1977 and 2003 energy content from added sugars in beverages increased by $377 \mathrm{~kJ} / \mathrm{d}(90 \mathrm{kcal} / \mathrm{d})$ and from added sugars in foods by $96 \mathrm{~kJ} / \mathrm{d}(23 \mathrm{kcal} / \mathrm{d})$ in the USA ${ }^{(3)}$. In spite of a decline in consumption of absolute energy content of added sugars, percentage of total energy intake from added sugars has remained high ${ }^{(3)}$. In light of the growing body of evidence highlighting unfavourable health effects of added sugars, the 2015 Dietary Guidelines Advisory Committee recommended that Americans limit sugar to no more than $10 \%$ of daily energy content ${ }^{(4)}$. Intake of high-fructose maize syrup or added sugars has been associated with the metabolic syndrome, characterised by elevated blood pressure, TAG, LDL-cholesterol, uric acid and inflammation ${ }^{(5-7)}$. Not surprisingly, there is some evidence that dietary added sugars are associated with cancer, although the evidence is limited. Case-control and prospective studies have shown an association of consumption of sugary foods ${ }^{(8,9)}$, and particularly beverages ${ }^{(10,11)}$, with increased risk of pancreatic cancer, which may be mediated in part through induction of transketolase ${ }^{(12)}$. In addition, sweet foods and beverages were shown to increase breast cancer risk by $27 \%{ }^{(13)}$. Recently, we reported an association between sugary beverages (fruit juices and sugar-sweetened) and reduced survival among head and neck cancer patients ${ }^{(14)}$.

Little is known about the associations of dietary added and concentrated sugars with the development of prostate cancer, although it is understood that lifestyle plays an important role in prostate cancer prevention ${ }^{(15)}$. Because of the putative link between chronic inflammation and prostate cancer, dietary items that are potentially pro-inflammatory deserve particular attention. It has been shown, for example that heterocyclic amines promote the development of cancer and induce accumulation of inflammatory cells (lymphocytes and macrophages) in the prostate ${ }^{(16)}$, that processed meat or dietary fat from meat is associated with increased prostate cancer risk ${ }^{(17,18)}$ and there is some evidence that dairy consumption may be associated with increased prostate cancer risk ${ }^{(19)}$. It is possible that the increased fructose and consequently TAG in items with concentrated sugars promotes an inflammatory response that supports DNA damage and genetic changes leading to neoplastic lesions of the prostate ${ }^{(6,20-22)}$. We hypothesised that consumption of sugar-dense items, or items with concentrated

Abbreviations: DHQ, diet history questionnaire; HR, hazard ratio; PLCO, Prostate, Lung, Colorectal and Ovarian; PSA, prostate-specific antigen.

* Corresponding author: F. L. Miles, fax +1 206667 7850, email fmiles@fredhutch.org 
sugars lacking the phytonutrients and fibre found in plant-based foods is particularly problematic, with a more detrimental impact on blood sugar, and ultimately promoting inflammation and prostate cancer growth.

The goal of the present study was to examine the association of concentrated sugars with prostate cancer risk. The term concentrated sugars was defined as sugars (in grams) from sugarsweetened beverages and fruit juices as well sugars in refined and processed desserts, constituting at least $30 \%$ of total energy content. Thus, this included added sugars in beverages and dessert foods, as well as natural sugars in fruit juices, which are naturally present in high amounts. These associations were evaluated in men receiving usual medical care in the prospective, population-based Prostate, Lung, Colorectal and Ovarian (PLCO) Cancer Screening Trial.

\section{Methods}

\section{Study population}

This study utilised data from the PLCO Cancer Screening Trial, a large, prospective, randomised, multi-site study (Birmingham, AL; Denver, CO; Detroit, MI; Honolulu, HI; Marshfield, WI; Minneapolis, MN; Pittsburgh, PA; Salt Lake City, UT; St Louis, MO and Washington, DC) designed initially to examine the effects of cancer screening on cancer mortality ${ }^{(23)}$. In brief, ten screening centres across the USA enrolled men ages 55-74 years to an intervention (screening) or control arm between 1993 and 2001. These men were recruited from the general population in the geographic area of the screening centres. A total of 38343 men were randomised to the control arm, where they received usual medical care from their health care providers, unlike men in the screening arm who received digital rectal exams and annual blood draws for prostate-specific antigen (PSA). The follow-up rate was $99 \cdot 5 \%$ (Fig. 1).

Annual study update questionnaires were used to ascertain prostate cancer incidence and were sent to all study participants. For men reporting a prostate cancer diagnosis, or men with abnormal test results from screening, medical records were obtained and used to confirm the diagnosis, clinical stage and grade. In the present study, cancers of Gleason $<7$ were defined as low grade, and cancers of Gleason $\geq 7$ were defined as high grade.

Of the 38343 men in the usual-care arm, 26927 completed the baseline and diet history questionnaires (DHQ). After excluding participants who were diagnosed with any cancer before the questionnaires (1867), skipped eight or more food frequency questions (1728), or were in the first or last percentile of energetic intake $(<2314 \mathrm{~kJ} / \mathrm{d}(<553 \mathrm{kcal} / \mathrm{d})$ and $>23510 \mathrm{~kJ} / \mathrm{d}$ $(>5619 \mathrm{kcal} / \mathrm{d})$ ) (1148), there were 23839 men in the eligible DHQ cohort. Additional exclusion of men with missing data for smoking status, pack-years of smoking, education, family history and diabetes history, which was found to be related to prostate cancer risk in this cohort ${ }^{(24)}$, resulted in an eligible cohort containing 22720 men. Median follow-up for these men was 9 years (183 430 person-years) (Fig. 1).

The study was reviewed and approved by the National Cancer Institute (NCI) institutional review board and screening centres (Clinicaltrials.gov identifier: NCT00002540).

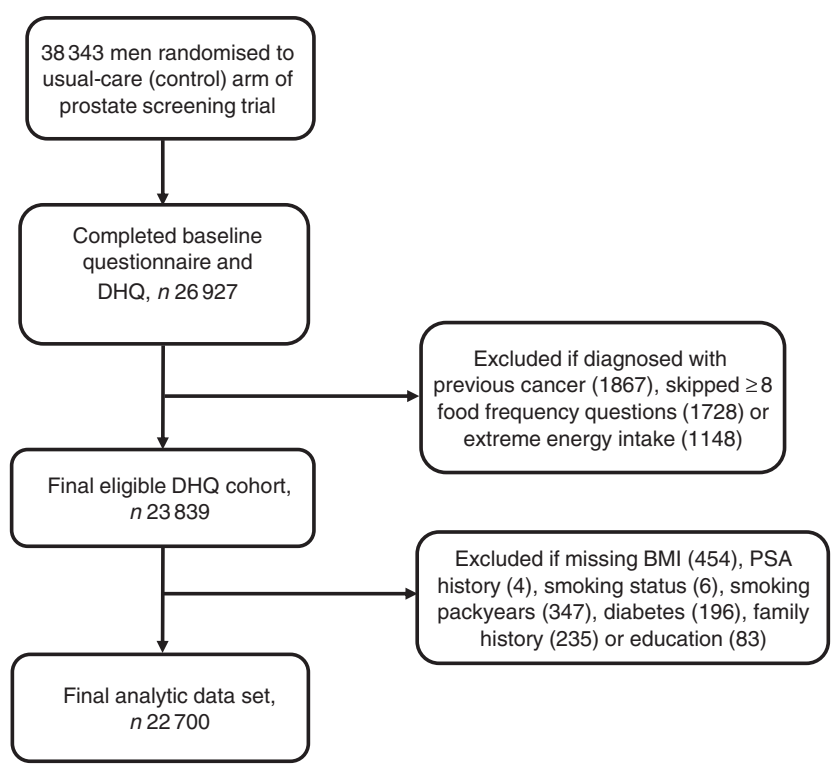

Fig. 1. Study design and flowchart of participant selection. DHQ, diet history questionnaire; PSA, prostate-specific antigen.

All procedures performed in studies involving human participants were in accordance with the ethical standards of the institutional and/or national research committee and with the 1964 Helsinki Declaration and its later amendments or comparable ethical standards.

\section{Data collection}

PLCO study participants completed a baseline questionnaire with information on demographics, medical history and smoking history. Height and weight were measured at the randomisation clinic visit and BMI was computed as weight $(\mathrm{kg}) /$ height $\left(\mathrm{m}^{2}\right)$. The DHQ, a FFQ developed by the Risk Factor Monitoring and Methods Branch of the NCI, was introduced in December of 1998, 5 years into the trial. It included 156 questions on frequencies of consumption of various foods and beverages from which daily nutrient intake data were estimated. The food list and nutrient database used with the DHQ are based on national dietary data (US Department of Agriculture's 1994-1996 Continuing Survey of Food Intakes by Individuals). The DHQ has been validated and found to be as good as or superior to two widely used FFQ at the time the PLCO study was conducted ${ }^{(25)}$. Participants in the usual-care arm randomised before 1998 were offered the DHQ in 1999 or 2000, around the anniversary of randomisation, and individuals randomised after December 1998 were offered the DHQ at baseline. The FFQ was self-administered and asked about frequency of consumption of desserts, sweetened beverages, fruit drinks, fruit juices, fruit, vegetables and other items. For the purposes of this analysis, sugars from soft drinks and sodas, milkshakes, punch, and fruit drinks, and sugar or honey added to tea or coffee were summed to generate a composite variable for sugar-sweetened beverages. Sugars from fruit juices included sugars from orange, grapefruit, tomato, and 'other' fruit or vegetable juices. In addition, we considered common desserts 
determined to have concentrated sugars (comprising at least $30 \%$ of total energy content per item), to generate a composite variable including sugars from cakes, cookies, pies, pastries, chocolate, candy, pudding, syrups, ice cream, and added sugar or sweet creams. In addition, the sum of all added sugars was calculated, including sugar added to processed foods or used in baked goods or sodas and other beverages, in addition to sugar added 'at the table'. The total amount of sugars in grams was calculated from the sum of fructose, galactose, glucose, lactose, maltose and sucrose using DietCalc Software developed by the NCI. The sugar variables were generated based on the Nutrition Data Systems for Research - Nutritional Analysis Software developed at the University of Minnesota.

\section{Statistical analysis}

The main categories of added sugar considered for analyses were sugar-sweetened beverages, fruit juices and desserts. In addition, composite variables representing the sum of these three variables, as well as intake of all added sugars from the diet were generated. The association of daily consumption of concentrated sugars with prostate cancer risk was analysed using the quartile distribution for sugar consumption for the final eligible DHQ cohort. Cox proportional hazards regression was used to calculate hazard ratios (HR) and 95\% CI. Sugar consumption was additionally modelled linearly, as a continuous variable, representing a ten-unit increment in dietary intakes. Follow-up time was calculated as the interval between days from completion of the DHQ to prostate cancer diagnosis, death, or 30 September 2009, depending on which came first. Models were adjusted for study centre, age, race (White $v$. nonWhite), education (less than high school, high school graduate, post-high school/some college, college graduate or more), cigarette status (never, current, former), pack-years of smoking, current BMI (at baseline), previous history of diabetes (yes/no), family history of prostate cancer (yes/no), number of PSA screens over the previous 3 years (none, once, more than once, unknown), and energy $(\mathrm{kJ} / \mathrm{d}(\mathrm{kcal} / \mathrm{d}))$. These variables were included as they possibly confound the relationship between sugar consumption and prostate cancer risk, or were previously found to be associated with prostate cancer risk. Other variables considered were red and processed meat $(\mathrm{g} / \mathrm{d})$, fruit (servings/d), and vegetables (servings/d), but were not included in final models as they were found to be non-influential on results. $P$ value for trend was calculated treating the exposure of interest as a continuous variable, based on the Wald statistic.

\section{Results}

Baseline characteristics of the eligible study cohort according to intake of concentrated sugars from foods and beverages are shown in Table 1. Differences were noted for previous history of diabetes, with the most individuals with a previous history $(47.8 \%)$ falling in the lowest quartile of sugar intake. Notable differences were also observed for race, with an increased percentage of Black participants in the highest quartile of intake of concentrated sugars (40.9\%), and more Asians in the lowest quartile (40\%) relative to the other quartiles. An increased proportion of men with high school or less education (approximately 29\%), as well as current smokers (approximately $32 \%$ ) were in the highest quartile of concentrated sugar consumption. A lower proportion of men in the highest quartile had multiple PSA screens (21.3\%). Participants in the highest quartile of concentrated sugar consumption consumed the greatest amounts of sugar from sugar-sweetened beverages (which were the major source of concentrated sugars), fruit juices and desserts, as well as added sugars overall. Energetic intake was notably higher for men in the highest quartile $(10669 \mathrm{~kJ} / \mathrm{d}(2550 \mathrm{kcal} / \mathrm{d}))$.

Multivariable-adjusted associations of consumption of concentrated sugars with prostate cancer risk are shown in Table 2. Consumption of sugars from sugar-sweetened beverages was associated with increased overall prostate cancer risk, with $21 \%$ increased risk for men in the top quartile of consumption (HR: $1.21 ; 95 \%$ CI 1.06, 1.39; $P_{\text {for trend }}<0.01$ ). In addition, an association of sugar-sweetened beverages with increased risk of low grade prostate cancer was observed for individuals in the highest quartile $(P=0 \cdot 02)$. However, there was no statistical difference between sugar consumption and risk of low and high grade prostate cancer. Consumption of sugars from fruit juices was associated with increased overall prostate cancer risk in the upper second and third quartiles, but the association diminished thereafter. There were no significant associations between consumption of sugars from desserts and prostate cancer risk. There was additionally no association between consumption of concentrated sugars and prostate cancer risk when sugar consumption was analysed as a continuous variable (not shown).

There were no associations between servings of sugarsweetened beverages and prostate cancer risk. There was also no discernable pattern between servings of fruit juices and prostate cancer risk (data not shown).

We sought to determine if the associations between sugars from sugar-sweetened beverages and prostate cancer risk were modified by race (White $v$. non-White) or number of PSA screens (none $v$. at least one) (Table 3). Number of PSA screens had no effect on the association between sugar consumption and prostate cancer risk, and there was no statistical interaction (Q4 v. Q1, $\left.P_{\text {interaction }}=0.92\right)$. Similarly, race did not significantly modify the association between consumption of sugars from sugar-sweetened beverages and prostate cancer risk (Q4v. Q1, $\left.P_{\text {interaction }}=0.94\right)$, and positive associations with sugar consumption were noted for White men only, in the third and fourth quartiles. As diabetes history has previously been inversely associated with prostate cancer risk, and is likely associated with sugar consumption, we also examined the association between sugar-sweetened beverages and prostate cancer risk in individuals without previous history of diabetes. The trend was similar to that seen in the full analytic cohort (Q2, HR: 1.11 ; $95 \%$ CI 0.97, 1.26; Q3, HR: 1.16; $95 \%$ CI 1.02, 1.33; Q4, HR: $1 \cdot 20 ; 95 \%$ CI 1.05, 1.37) (not shown).

We additionally examined multivariable-adjusted associations between consumption of all concentrated sugars (sugarsweetened beverages, fruit juices and desserts) or all dietary added sugars and prostate cancer risk (Table 4). No significant associations were found. 
Table 1. Baseline, demographic and lifestyle characteristics of participants in the control arm of the Prostate, Lung, Colorectal and Ovarian study according to intake of concentrated sugars ${ }^{\star} \dagger$ (Numbers and percentages; mean values and standard deviations)

\begin{tabular}{|c|c|c|c|c|c|c|c|c|c|c|}
\hline & & & \multicolumn{8}{|c|}{ Quartiles (g/d) } \\
\hline & \multicolumn{2}{|c|}{ Total } & \multicolumn{2}{|c|}{$<23.47(n 5680)$} & \multicolumn{2}{|c|}{$23 \cdot 47-40 \cdot 20(n 5680)$} & \multicolumn{2}{|c|}{$40 \cdot 21-65.93(n 5678)$} & \multicolumn{2}{|c|}{$\geq 65.94$ (n 5682) } \\
\hline & $n$ & $\%$ & $n$ & $\%$ & $n$ & $\%$ & $n$ & $\%$ & $n$ & $\%$ \\
\hline Family history & 1625 & $7 \cdot 2$ & 359 & $22 \cdot 1$ & 386 & 23.8 & 443 & $27 \cdot 3$ & 437 & $26 \cdot 9$ \\
\hline History of diabetes & 1842 & $8 \cdot 1$ & 880 & $47 \cdot 8$ & 473 & $25 \cdot 7$ & 282 & $15 \cdot 3$ & 207 & 11.2 \\
\hline \multicolumn{11}{|l|}{ Ethnicity } \\
\hline White & 20633 & $90 \cdot 8$ & 5040 & 24.4 & 5190 & $25 \cdot 2$ & 5251 & $25 \cdot 5$ & 5152 & $25 \cdot 0$ \\
\hline Black & 589 & $2 \cdot 6$ & 103 & 17.5 & 114 & $19 \cdot 4$ & 131 & $22 \cdot 2$ & 241 & $40 \cdot 9$ \\
\hline Hispanic & 357 & 1.6 & 90 & $25 \cdot 2$ & 76 & 21.3 & 93 & $26 \cdot 1$ & 98 & 27.5 \\
\hline Asian/Pacific Islander & 1089 & 4.8 & 436 & $40 \cdot 0$ & 286 & $26 \cdot 3$ & 192 & $17 \cdot 6$ & 175 & $16 \cdot 1$ \\
\hline Other & 52 & 0.2 & 11 & $21 \cdot 2$ & 14 & 26.9 & 11 & $21 \cdot 2$ & 16 & $30 \cdot 8$ \\
\hline \multicolumn{11}{|l|}{ Education } \\
\hline$<12$ years & 1542 & $6 \cdot 8$ & 394 & $25 \cdot 6$ & 319 & 20.7 & 384 & 24.9 & 445 & 28.9 \\
\hline High school & 4187 & $25 \cdot 3$ & 1005 & 24.0 & 988 & 23.6 & 990 & 23.6 & 1204 & 28.8 \\
\hline Post-high school & 7392 & 57.9 & 1885 & 25.5 & 1786 & 24.2 & 1807 & 24.5 & 1914 & 25.9 \\
\hline College graduate & 9557 & $42 \cdot 1$ & 2383 & 24.9 & 2577 & $27 \cdot 0$ & 2484 & $26 \cdot 0$ & 2113 & $22 \cdot 1$ \\
\hline \multicolumn{11}{|l|}{ Smoking } \\
\hline Never & 8588 & 37.7 & 1881 & 22.0 & 2090 & 24.4 & 2232 & $26 \cdot 1$ & 2355 & 27.5 \\
\hline Current & 2301 & $10 \cdot 1$ & 520 & $22 \cdot 6$ & 499 & 21.7 & 551 & $24 \cdot 0$ & 731 & 31.8 \\
\hline Former & 11861 & $52 \cdot 2$ & 3279 & $27 \cdot 7$ & 3091 & $26 \cdot 1$ & 2895 & $24 \cdot 4$ & 2596 & 21.9 \\
\hline \multicolumn{11}{|l|}{ Number of PSA screens $\ddagger$} \\
\hline None & 9956 & 43.4 & 2430 & 24.4 & 2401 & 24.1 & 2444 & $24 \cdot 6$ & 2681 & $26 \cdot 9$ \\
\hline Once & 8518 & 37.5 & 2135 & $25 \cdot 1$ & 2167 & $25 \cdot 4$ & 2187 & $25 \cdot 7$ & 2029 & 23.8 \\
\hline More than once & 2314 & $10 \cdot 2$ & 586 & $25 \cdot 3$ & 637 & 27.5 & 598 & $25 \cdot 8$ & 493 & 21.3 \\
\hline \multirow[t]{2}{*}{ Unknown } & 1932 & 8.5 & 529 & $27 \cdot 4$ & 475 & $24 \cdot 6$ & 449 & $23 \cdot 2$ & 479 & 24.8 \\
\hline & Mean & SD & Mean & SD & Mean & SD & Mean & SD & Mean & SD \\
\hline Age (years) & $65 \cdot 6$ & 5.9 & 65.5 & 5.8 & 65.9 & 5.9 & 65.9 & $6 \cdot 0$ & 65.0 & 6.0 \\
\hline Pack-years & 24.4 & $30 \cdot 8$ & $26 \cdot 6$ & 30.9 & $24 \cdot 1$ & 30.3 & 23.0 & $20 \cdot 1$ & 23.6 & $31 \cdot 7$ \\
\hline BMI $\left(\mathrm{kg} / \mathrm{m}^{2}\right)$ & $27 \cdot 5$ & $4 \cdot 1$ & 27.9 & 4.3 & 27.5 & 3.9 & $27 \cdot 3$ & 4.0 & 27.5 & 4.1 \\
\hline Sugar-sweetened beverages $(\mathrm{g} / \mathrm{d})$ & $16 \cdot 0$ & $29 \cdot 7$ & $2 \cdot 0$ & 3.2 & 5.8 & 6.8 & $12 \cdot 5$ & $12 \cdot 4$ & 43.7 & $47 \cdot 2$ \\
\hline Fruit juices (g/d) & 13.7 & $19 \cdot 6$ & 4.6 & 4.5 & $10 \cdot 4$ & 7.9 & 14.9 & 11.9 & 24.8 & 33.0 \\
\hline Desserts $(\mathrm{g} / \mathrm{d})$ & 21.5 & $20 \cdot 1$ & 7.7 & $5 \cdot 1$ & $15 \cdot 4$ & 8.5 & $24 \cdot 2$ & 13.3 & 38.9 & $28 \cdot 2$ \\
\hline All added sugars (teaspoons/d) & $14 \cdot 3$ & $10 \cdot 3$ & $6 \cdot 4$ & 3.1 & $10 \cdot 0$ & 3.6 & 14.4 & 4.5 & $26 \cdot 4$ & $12 \cdot 6$ \\
\hline Energy content (kJ/d) & 8322 & 3414 & 6581 & 2883 & 7464 & 2753 & 8577 & 2916 & 10669 & 3586 \\
\hline Energy content $(\mathrm{kcal} / \mathrm{d})$ & 1989 & 816 & 1573 & 689 & 1784 & 658 & 2050 & 697 & 2550 & 857 \\
\hline
\end{tabular}

* Quartiles represent total sugars consumed from sugar-sweetened beverages, fruit juices and desserts.

$\dagger P$ value family history $=0.01 ; P$ value for all other variables $<0.0001$

† Screening over previous 3 years. 
Table 2. Multivariable associations of consumption of concentrated sugars $(\mathrm{g})$ from beverages with prostate cancer risk $† \ddagger$ (Hazard ratios (HR) and $95 \%$ confidence intervals)

\begin{tabular}{|c|c|c|c|c|c|c|c|c|c|}
\hline & \multicolumn{3}{|c|}{ Sugar-sweetened beverages } & \multicolumn{3}{|c|}{ Fruit juices } & \multicolumn{3}{|c|}{ Desserts } \\
\hline & $\begin{array}{l}\text { Cases }(n) / \\
\text { total person-years }\end{array}$ & $\mathrm{HR}$ & $95 \% \mathrm{Cl}$ & $\begin{array}{l}\text { Cases }(n) / \\
\text { total person-years }\end{array}$ & $\mathrm{HR}$ & $95 \% \mathrm{Cl}$ & $\begin{array}{c}\text { Cases }(n) / \\
\text { total person-years }\end{array}$ & HR & $95 \% \mathrm{Cl}$ \\
\hline \multicolumn{10}{|c|}{ Prostate cancer ( $n$ 1996) } \\
\hline Q1 & $398 / 41955$ & 1.00 & - & $433 / 45505$ & 1.00 & - & $481 / 45662$ & 1.00 & - \\
\hline Q2 & $544 / 48923$ & $1 \cdot 11$ & $0.97,1.26$ & $524 / 46618$ & $1 \cdot 14$ & $1.01,1.30$ & $488 / 45975$ & 0.97 & $0.85,1.10$ \\
\hline Q3 & $519 / 45944$ & $1 \cdot 14$ & $1.00,1.31$ & $514 / 44009$ & $1 \cdot 15$ & $1.01,1.31$ & $527 / 45746$ & 1.03 & $0.91,1.18$ \\
\hline Q4 & $535 / 46608$ & $1 \cdot 21^{\star *}$ & $1.06,1.39$ & $525 / 47298$ & 1.07 & $0.94,1.22$ & $500 / 46047$ & 0.95 & $0.83,1.10$ \\
\hline \multicolumn{10}{|c|}{ Low grade $(n 1014)$} \\
\hline Q1 & $202 / 41032$ & 1.00 & - & $218 / 44466$ & 1.00 & - & $262 / 44596$ & 1.00 & - \\
\hline Q2 & $274 / 47668$ & 1.09 & $0.91,1.31$ & $271 / 45336$ & $1 \cdot 17$ & $0.97,1.39$ & $241 / 44751$ & 0.86 & $0.72,1.03$ \\
\hline Q3 & $251 / 44693$ & $1 \cdot 14$ & $0.94,1.37$ & $262 / 42822$ & $1 \cdot 20$ & $0.95,1.37$ & $257 / 44436$ & 0.90 & $0.76,1.08$ \\
\hline Q4 & $277 / 45305$ & $1 \cdot 25^{\star}$ & $1.03,1.51$ & $263 / 46074$ & 1.05 & $0.87,1.26$ & $254 / 44915$ & 0.87 & $0.71,1.05$ \\
\hline \multicolumn{10}{|c|}{ High grade ( $n 960)$} \\
\hline Q1 & $193 / 41020$ & 1.00 & - & $212 / 44534$ & 1.00 & - & $217 / 44493$ & 1.00 & - \\
\hline Q2 & $264 / 47746$ & $1 \cdot 12$ & $0.93,1.36$ & $248 / 45378$ & $1 \cdot 13$ & $0.94,1.36$ & $244 / 44973$ & $1 \cdot 10$ & $0.91,1.33$ \\
\hline Q3 & $251 / 44850$ & $1 \cdot 14$ & $0.94,1.39$ & $243 / 42915$ & $1 \cdot 13$ & $0.94,1.36$ & $262 / 44563$ & $1 \cdot 18$ & $0.98,1.43$ \\
\hline Q4 & $252 / 45333$ & $1 \cdot 19$ & $0.97,1.45$ & $257 / 46122$ & 1.09 & $0.90,1.31$ & $237 / 44920$ & 1.05 & $0.85,1.29$ \\
\hline
\end{tabular}

Q, quartile; PSA, prostate-specific antigen

${ }^{*} P_{\text {for trend }}<0.05,{ }^{\star *} P_{\text {for trend }}<0.01$.

$\dagger$ Adjusted for age, race, study centre, BMI, education, smoking, family history of prostate cancer, history of diabetes, PSA screening and energy intake.

‡ Quartile cutpoints $(\mathrm{g} / \mathrm{d})$ : sugar-sweetened beverages - 0.63, 4.83, 19.21; fruit juices $-2.55,9 \cdot 24,20 \cdot 01$; desserts - 7.85, $16 \cdot 18,28 \cdot 87$.

Table 3. Multivariable associations of consumption of concentrated sugars $(\mathrm{g})$ from sugar-sweetened beverages with prostate cancer risk according to prostate-specific antigen (PSA) screens and race* $\dagger$

(Hazard ratios (HR) and $95 \%$ confidence intervals)

\begin{tabular}{|c|c|c|c|c|c|c|c|}
\hline & \multicolumn{3}{|c|}{ No PSA screens } & \multicolumn{3}{|c|}{$\geq 1$ PSA screen } & \multirow[b]{2}{*}{$P \neq$} \\
\hline & Cases $(n) /$ total person-years & $\mathrm{HR}$ & $95 \% \mathrm{Cl}$ & Cases $(n) /$ total person-years & $\mathrm{HR}$ & $95 \% \mathrm{Cl}$ & \\
\hline Q1 & $180 / 20059$ & 1.00 & - & $216 / 24553$ & 1.00 & - & \\
\hline Q2 & $225 / 20123$ & $1 \cdot 16$ & $0.95,1.41$ & $253 / 23447$ & 1.04 & $0.86,1.24$ & 0.40 \\
\hline Q3 & 219/20209 & $1 \cdot 15$ & $0.94,1.40$ & $271 / 21526$ & $1 \cdot 15$ & $0.97,1.38$ & 0.95 \\
\hline \multirow[t]{2}{*}{ Q4 } & $218 / 20511$ & $1 \cdot 18$ & $0.97,1.44$ & $271 / 17630$ & $1 \cdot 17$ & $0.97,1.40$ & 0.92 \\
\hline & \multicolumn{3}{|c|}{ White } & \multicolumn{3}{|c|}{ Non-white } & \\
\hline Q1 & $368 / 39120$ & 1.00 & - & $38 / 2835$ & 1.00 & - & \\
\hline Q2 & $448 / 40082$ & 1.22 & $0.98,1.29$ & $46 / 3365$ & 1.07 & $0.70,1.63$ & 0.83 \\
\hline Q3 & $488 / 41140$ & 1.20 & $1.04,1.37$ & $34 / 4566$ & 0.78 & $0.49,1.22$ & 0.07 \\
\hline Q4 & $513 / 46084$ & 1.19 & $1 \cdot 03,1 \cdot 36$ & $61 / 6238$ & $1 \cdot 21$ & $0.80,1.82$ & 0.94 \\
\hline
\end{tabular}

Q, quartile.

* Adjusted for age, race, study centre, BMI, education, smoking, family history of prostate cancer, history of diabetes, PSA screening and energy intake.

† Quartile cutpoints (g/d): no PSA screens - 0.89, 5.95, 21.17; $\geq 1$ PSA screen - 0.63, 4.13, 17.23; white - 0.63, 3.92, 16.42; non-white - 1.19, 7.74, 22.67.

$\ddagger P$ value from heterogeneity test.

\section{Discussion}

In this prospective study of men within the usual-care arm of the PLCO screening trial, we found that consumption of sugars from sugar-sweetened beverages was associated with increased prostate cancer risk for men in the upper third and fourth quartiles of sugar intake.

We did not find a similar trend with consumption of sugars from fruit juices. There was no linear association even after controlling for grams of tomato and vegetable juice consumed or exclusion of tomato and vegetable juices from the exposure variable. Type of fruit juice consumed might be particularly relevant, but this is not discernible from the present study. Additional investigation revealed $<30 \%$ overlap for consumption of sugars from fruit-juices and sugar-sweetened beverages comparing any one quartile, suggesting the association of consumption of fruit juices with prostate cancer risk was not confounded by sugar-sweetened beverages and vice versa.

Number of servings of sugar-sweetened beverages overall was low, and it was not possible to examine the quartile distribution of servings of these beverages without including servings of sugars added to tea and coffee, which included sugar and honey, which likely confounded results. Tea and coffee contain polyphenols and can potentially confound the association of sugar-rich beverages with prostate cancer risk. Moreover, it was not possible to separate sugars and honey added to tea or coffee. When analysing sugars in grams of soft drinks only, excluding sugars added to tea and coffee, the association was still present but not as strong, and the linear 
Table 4. Multivariable associations of all concentrated or non-natural sugars with prostate cancer risk*

(Hazard ratios (HR) and $95 \%$ confidence intervals)

\begin{tabular}{lccc}
\hline Quartiles & Cases $(n)$ /total person-years & $\mathrm{HR}$ & $95 \% \mathrm{Cl}$ \\
\hline All concentrated + & & & \\
Q1 & $451 / 45684$ & 1.00 & - \\
Q2 & $525 / 45579$ & $1 \cdot 11$ & $0.97,1.26$ \\
Q3 & $507 / 46021$ & 1.04 & $0.91,1.19$ \\
Q4 & $513 / 46146$ & 1.07 & $0.93,1 \cdot 23$ \\
All added & & & \\
Q1 & $457 / 45316$ & 1.00 & - \\
Q2 & $535 / 45766$ & $1 \cdot 12$ & $0.99,1.28$ \\
Q3 & $488 / 45967$ & 1.00 & $0.88,1.15$ \\
Q4 & $516 / 46381$ & 1.08 & $0.93,1 \cdot 25$ \\
\hline
\end{tabular}

$\mathrm{Q}$, quartile; PSA, prostate-specific antigen.

* Adjusted for age, race, treatment centre, BMI, education, smoking, family history of prostate cancer, history of diabetes, PSA screening and energy intake.

$\dagger$ Includes desserts, sugar-sweetened beverages and fruit juices; quartile cutpoints $(\mathrm{g} / \mathrm{d}): 23 \cdot 47,40 \cdot 20,65 \cdot 94$.

‡ Quartile cutpoints (teaspoons/d): 7.43, 11.73, 18.15 .

trend persisted. This might be expected as grams of sugars added to tea and coffee constituted approximately $25 \%$ of sugars in the sugar-sweetened beverages category.

Other studies have examined the association between carbohydrate or added sugar intake and prostate cancer risk. In a prospective Swedish cohort study, it was found that some refined carbohydrates including cakes and biscuits, low-fibre cereals and rice and pasta were associated with low-grade or overall prostate cancer $^{(26)}$. Increased consumption of sugar-sweetened beverages was associated with increased risk of symptomatic prostate cancer, characterised by malignancy-related symptoms (but not total or low-grade cancer). In a separate prospective study, an inverse association was reported between total fructose intake (fruit and non-fruit-derived) and prostate cancer risk ${ }^{(27)}$. Fructose, however, has been shown to promote growth of pancreatic cancer cells, and is believed to have an important role in inflammation $^{(6,12)}$. We did not observe an association between fructose and prostate cancer risk in our study (not shown). Neither was an association observed when considering a composite variable representing all added sugars from the diet. These findings emphasise the potential significance of concentrated added sugars on prostate cancer risk. In our study, sugarsweetened beverages, and particularly sodas, contributed the greatest amount of sugar relative to the other categories.

Other dietary and behavioural factors may affect prostate cancer risk. Fruit and vegetable consumption has been shown to affect cancer risk, although evidence on the relative role of fruit and vegetables in prostate cancer susceptibility is inconclusive. It was previously reported that high vegetable consumption may be associated with reduced risk of aggressive prostate cancer in the PLCO study ${ }^{(28)}$. In our models, the effect of daily servings of vegetables and fruits on associations between sugar intake and prostate cancer risk was negligible.

It is plausible that body weight might interact with sugar to increase prostate cancer risk, but the relationship between obesity and prostate cancer is complex. There is evidence that obese men may have higher risk of high-grade cancers, although they may have lower PSA levels ${ }^{(29-31)}$. In addition, increased intake of sugar-sweetened beverages has been associated with increased weight gain ${ }^{(32,33)}$, although an inverse association between BMI and sugar intake has also been reported for men, particularly ${ }^{(34)}$. For this reason BMI was included as a confounder when analysing the association between dietary sugar and prostate cancer risk, although its effect on estimates was negligible. Comparisons at baseline revealed markedly increased energy intake for men in the highest quartile of sugar consumption, although mean BMI was not higher for these men. Regular or vigorous exercise might also be associated with reduced risk of prostate cancer, particularly advanced cancer, based on some evidence from large, prospective cohort studies ${ }^{(35-37)}$. It is unclear how physical activity affected the association between sugar consumption and prostate cancer risk in the usual-care arm, as this information was not ascertainable from the DHQ.

Consumption of concentrated sugars may be related to prostate cancer risk through activation of inflammatory cytokines, such as interleukins, C-reactive protein, and $\mathrm{TNF}^{(38)}$, among others, as a result of elevated uric acid in the serum or another mechanism. Increases in uric acid, particularly, may lead to increased production of IL- $1 \beta$, and chronic inflammation $^{(39)}$. Alternatively, elevated TAG or cholesterol could be related to prostate cancer risk ${ }^{(40)}$ by inducing activation of signalling by $\mathrm{NF}-\kappa \mathrm{B}^{(21)}$ or protein kinase $\mathrm{B}$, and ultimately other inflammatory factors ${ }^{(41,42)}$. Furthermore, there is evidence that fructose, a common compound present at high concentrations in sugar-sweetened beverages and desserts, is converted to fat more rapidly than other sugars ${ }^{(43)}$. It is true that naturally occurring sugars and added sugars share the same chemical structure. However, the difference to be noted is perhaps in the broader range of physiological effects that ultimately regulate inflammatory processes. It is important to highlight that processed sugar-dense goods may not have the same effect on the blood glucose. Absorption of sugars in plant foods occurs more slowly and is more regulated due to buffering by vitamins and fibre, and phytonutrients, such that there is not a spike in blood sugar and consequently, a heightened inflammatory response ${ }^{(44)}$. Therefore, studies examining consumption of total sugars may not report positive associations with chronic disease. In addition to the potentially greater relevance of concentrated sugars over total sugars, our study highlights a more detrimental role of concentrated sugars from beverages than desserts in the context of prostate cancer risk. Physiological events associated with digestion and metabolism of these sugars, particularly, could lead to increases or alterations in proinflammatory cytokines, and ultimately chronic inflammation.

Strengths of the study include the large number of study participants and high follow-up rate, detailed information on sugar consumption obtained from the FFQ and subsequently thorough nutrient analysis, as well as complete information on demographic and clinical factors affecting prostate cancer risk. Limitations include possible measurement error in sugar intake due to misreporting or information bias, and limitations of the nutrient database in distinguishing natural from added sugars. Also, because dietary habits fluctuate, interpretation of findings of diet-related associations with cancer is more difficult in studies where the exposure assessed represents a single point in time. We were unable to accurately compare the role of sugar 
consumption with prostate cancer risk between the screening and usual-care arms of the PLCO, as differences in the dietary assessment tools between study arms as well as the timing of administration of the DHQ precluded direct comparison. Furthermore, while risk of advanced stage prostate cancer or prostate cancer mortality are important clinically relevant endpoints, we were unable to examine the association of sugar consumption with these endpoints due to the very low number of men with advanced stage prostate cancer and limited number of prostate cancer-specific deaths. Therefore, conclusions of the present study are limited and consumption of concentrated sugars should be examined in other settings.

In conclusion, this study provides evidence for a positive association between sugars from sugar-sweetened beverages and increased risk of prostate cancer among men receiving usual medical care in the PLCO trial. Our findings highlight the potential significance of high consumption of added, concentrated sugars from beverages in prostate cancer aetiology. Additional studies examining this association are warranted.

\section{Acknowledgements}

The authors would like to thank the National Cancer Institute for access to data collected in the Prostate, Lung, Colorectal and Ovarian Cancer Screening Trial. The authors declare that the statements contained herein are solely those of the authors and do not represent or imply concurrence or endorsement by the National Cancer Institute.

This research was partially supported by the National Institutes of Health (Z. F. Z. and F. L. M., T32 CA09142), (F. L. M. and M. L. N., R25 CA092408); and the Alper Research Funds for Environmental Genomics of the UCLA Jonsson Comprehensive Cancer Center (Z. F. Z.).

F. L. M. was responsible for writing and preparing the manuscript, developing the research questions and analysing data. M. L. N. and Z. F. Z. assisted in the statistical design, analytical approach and interpretation of data, and provided significant manuscript edits

The authors declare that there are no conflicts of interest.

\section{References}

1. Popkin BM \& Nielsen SJ (2003) The sweetening of the world's diet. Obes Res 11, 1325-1332.

2. United States Department of Agriculture, Office of Communications (2001) Profiling food consumption in America. In Agriculture Fact Book 2001-2002, pp. 13-22. Washington, DC: Superintendent of Documents, US Government Printing Office.

3. Powell ES, Smith-Taillie LP \& Popkin BM (2016) Added sugars intake across the distribution of US children and adult consumers: 1977-2012. I Acad Nutr Diet 116, 1543-1550.e1.

4. United States Department of Agriculture (2015) 2015-2020 Dietary Guidelines for Americans, 8th ed. Washington, DC: USDA

5. Johnson RJ, Perez-Pozo SE, Sautin YY, et al. (2009) Hypothesis: could excessive fructose intake and uric acid cause type 2 diabetes? Endocr Rev 30, 96-116.

6. Johnson RJ, Sanchez-Lozada LG \& Nakagawa T (2010) The effect of fructose on renal biology and disease. J Am Soc Nephrol 21, 2036-2039.
7. Jalal DI, Smits G, Johnson RJ, et al. (2010) Increased fructose associates with elevated blood pressure. J Am Soc Nephrol 21, 1543-1549.

8. Chan JM, Wang F \& Holly EA (2009) Sweets, sweetened beverages, and risk of pancreatic cancer in a large populationbased case-control study. Cancer Causes Control 20, 835-846.

9. Larsson SC, Bergkvist L \& Wolk A (2006) Consumption of sugar and sugar-sweetened foods and the risk of pancreatic cancer in a prospective study. Am J Clin Nutr 84, 1171-1176.

10. Mueller NT, Odegaard A, Anderson K, et al. (2010) Soft drink and juice consumption and risk of pancreatic cancer: the Singapore Chinese Health Study. Cancer Epidemiol Biomarkers Prev 19, 447-455.

11. Schernhammer ES, Hu FB, Giovannucci E, et al. (2005) Sugarsweetened soft drink consumption and risk of pancreatic cancer in two prospective cohorts. Cancer Epidemiol Biomarkers Prev 14, 2098-2105.

12. Liu H, Huang D, McArthur DL, et al. (2010) Fructose induces transketolase flux to promote pancreatic cancer growth. Cancer Res 70, 6368-6376.

13. Bradshaw PT, Sagiv SK, Kabat GC, et al. (2009) Consumption of sweet foods and breast cancer risk: a case-control study of women on Long Island, New York. Cancer Causes Control 20, 1509-1515.

14. Miles FL, Chang SC, Morgenstern H, et al. (2016) Association of sugary beverages with survival among patients with cancers of the upper aerodigestive tract. Cancer Causes Control 27, $1293-1300$

15. Heber DFS, Jones LW \& Nelson WG (2009) Nutrition, Exercise and Prostate Cancer. Santa Monica, CA: Prostate Cancer Foundation.

16. Nakai Y, Nelson WG \& De Marzo AM (2007) The dietary charred meat carcinogen 2-amino-1-methyl-6-phenylimidazo [4,5-b]pyridine acts as both a tumor initiator and promoter in the rat ventral prostate. Cancer Res 67, 1378-1384.

17. Cross AJ, Peters U, Kirsh VA, et al. (2005) A prospective study of meat and meat mutagens and prostate cancer risk. Cancer Res 65, 11779-11784.

18. Giovannucci E, Rimm EB, Colditz GA, et al. (1993) A prospective study of dietary fat and risk of prostate cancer. J Natl Cancer Inst 85, 1571-1579.

19. Gao X, LaValley MP \& Tucker KL (2005) Prospective studies of dairy product and calcium intakes and prostate cancer risk: a meta-analysis. J Natl Cancer Inst 97, 1768-1777.

20. De Marzo AM, Platz EA, Sutcliffe S, et al. (2007) Inflammation in prostate carcinogenesis. Nat Rev Cancer 7, 256-269.

21. Welty FK (2013) How do elevated triglycerides and low HDLcholesterol affect inflammation and atherothrombosis? Curr Cardiol Rep 15, 400.

22. Sfanos KS \& De Marzo AM (2012) Prostate cancer and inflammation: the evidence. Histopathology 60, 199-215.

23. Prorok PC, Andriole GL, Bresalier RS, et al. (2000) Design of the Prostate, Lung, Colorectal and Ovarian (PLCO) Cancer Screening Trial. Control Clin Trials 21, Suppl. 6, 273S-309S.

24. Leitzmann MF, Ahn J, Albanes D, et al. (2008) Diabetes mellitus and prostate cancer risk in the Prostate, Lung, Colorectal, and Ovarian Cancer Screening Trial. Cancer Causes Control 19, $1267-1276$.

25. Subar AF, Thompson FE, Kipnis V, et al. (2001) Comparative validation of the Block, Willett, and National Cancer Institute food frequency questionnaires:the Eating at America's Table Study. Am J Epidemiol 154, 1089-1099.

26. Drake I, Sonestedt E, Gullberg B, et al. (2012) Dietary intakes of carbohydrates in relation to prostate cancer risk: a prospective study in the Malmo Diet and Cancer cohort. Am J Clin Nutr 96, 1409-1418. 
27. Giovannucci E, Rimm EB, Wolk A, et al. (1998) Calcium and fructose intake in relation to risk of prostate cancer. Cancer Res 58, 442-447.

28. Kirsh VA, Peters U, Mayne ST, et al. (2007) Prospective study of fruit and vegetable intake and risk of prostate cancer. $J$ Natl Cancer Inst 99, 1200-1209.

29. Baillargeon J, Pollock BH, Kristal AR, et al. (2005) The association of body mass index and prostate-specific antigen in a population-based study. Cancer 103, 1092-1095.

30. Gong Z, Neuhouser ML, Goodman PJ, et al. (2006) Obesity, diabetes, and risk of prostate cancer: results from the prostate cancer prevention trial. Cancer Epidemiol Biomarkers Prev 15, 1977-1983.

31. MacInnis RJ \& English DR (2006) Body size and composition and prostate cancer risk: systematic review and metaregression analysis. Cancer Causes Control 17, 989-1003.

32. Malik VS, Schulze MB \& Hu FB (2006) Intake of sugarsweetened beverages and weight gain: a systematic review. Am J Clin Nutr 84, 274-288.

33. Te Morenga L, Mallard S \& Mann J (2012) Dietary sugars and body weight: systematic review and meta-analyses of randomised controlled trials and cohort studies. BMJ 346, e7492.

34. Macdiarmid JI, Vail A, Cade JE, et al. (1998) The sugar-fat relationship revisited: differences in consumption between men and women of varying BMI. Int $J$ Obes Relat Metab Disord 22, 1053-1061.

35. Giovannucci EL, Liu Y, Leitzmann MF, et al. (2005) A prospective study of physical activity and incident and fatal prostate cancer. Arch Intern Med 165, 1005-1010.
36. Nilsen TI, Romundstad PR \& Vatten LJ (2006) Recreational physical activity and risk of prostate cancer: a prospective population-based study in Norway (the HUNT study). Int $J$ Cancer 119, 2943-2947.

37. Orsini N, Bellocco R, Bottai M, et al. (2009) A prospective study of lifetime physical activity and prostate cancer incidence and mortality. Br J Cancer 101, 1932-1938.

38. Lyngdoh T, Marques-Vidal P, Paccaud F, et al. (2011) Elevated serum uric acid is associated with high circulating inflammatory cytokines in the population-based Colaus study. PLOS ONE 6, e19901.

39. Shi Y (2010) Caught red-handed: uric acid is an agent of inflammation. J Clin Invest 120, 1809-1811.

40. Allott EH, Howard LE, Cooperberg MR, et al. (2014) Serum lipid profile and risk of prostate cancer recurrence: results from the SEARCH database. Cancer Epidemiol Biomarkers Prev 23, 2349-2356.

41. Ramos-Nino ME (2013) The role of chronic inflammation in obesity-associated cancers. ISRN Oncol 2013, 697521.

42. Zhuang L, Lin J, Lu ML, et al. (2002) Cholesterol-rich lipid rafts mediate akt-regulated survival in prostate cancer cells. Cancer Res 62, 2227-2231.

43. Parks EJ, Skokan LE, Timlin MT, et al. (2008) Dietary sugars stimulate fatty acid synthesis in adults. J Nutr $\mathbf{1 3 8}$, 1039-1046.

44. Krebs-Smith SM, Cleveland LE, Ballard-Barbash R, et al. (1997) Characterizing food intake patterns of American adults. Am J Clin Nutr 65, Suppl. 4, 1264S-1268S. 